\title{
POLÍTICAS URBANAS PARA EL DESARROLLO Y LA INNOVACIÓN EN LAS CIUDADES INTERMEDIAS ${ }^{1}$
}

\author{
Henar Pascual Ruiz-Valdepeñas \\ José Luis García Cuesta \\ Departamento de Geografía \\ Universidad de Valladolid
}

\section{RESUMEN}

En los últimos años se asiste al fortalecimiento del papel de las ciudades intermedias al estimarse su capacidad para lograr una mayor cohesión económica, social y territorial en Europa. Las políticas territoriales se orientan a reforzar sus ventajas competitivas y a potenciar la configuración y consolidación de redes de cooperación en las que las ciudades actúan como partes de un modelo policéntrico. La valoración de los impactos de los programas europeos en las ciudades y el análisis de las políticas y estrategias urbanas, ponen de relieve la naturaleza específica de los diversos niveles de intervención pública en el territorio y la multiplicidad de las interacciones que se generan entre ellos. En el nivel local de intervención, la planificación estratégica amplía el abanico de objetivos y actuaciones y aporta nuevos instrumentos que ponen el acento en el reforzamiento de los factores de competitividad urbana.

Palabras clave: ciudades intermedias, redes urbanas de cooperación, desarrollo policéntrico, planificación estratégica, competitividad urbana.

\begin{abstract}
The role of intermediate cities has been strengthened in recent years, after having evaluated their capacity to achieve economic, social and territorial cohesion. Territorial policies are orientated towards reinforcing their competitive advantages and optimising the configuration and consolidation of cooperation networks in cities that form a part of a polycentric model. The assessment of the impact of European programmes on cities and the analysis of urban policies and strategies highlights the specific nature of
\end{abstract}

1 El presente artículo se ha realizado en el marco del proyecto del Plan Nacional de I+D+i Procesos de innovación en ciudades intermedias y desarrollo policéntrico en España (SEJ2006-14277-C04-04), que a su vez forma parte de un Proyecto Coordinado entre el Instituto de Economía, Geografía y Demografía (CSIC) y las Universidades de Lérida, Sevilla y Valladolid. El investigador principal del Proyecto Coordinado es Ricardo Méndez Gutiérrez del Valle. 
the different levels of public intervention in the territory and the interactions that occur between them. On a local level of intervention, strategic planning widens the spectrum of objectives and actions and provides new instruments that focus on strengthening urban competitiveness factors.

Key words: intermediate cities, urban cooperation networks, polycentric development, strategic planning, urban competitiveness.

\section{Introducción}

Durante los últimos años hemos asistido a un creciente interés por el estudio y la interpretación de los sistemas urbanos formados por ciudades intermedias, entendiendo por éstas las que desempeñan funciones de intermediación entre los territorios rurales y urbanos de su área de influencia y son capaces de crear relaciones y tejer redes de flujos - bienes, innovación, información... - entre los espacios locales y los espacios regionales y nacionales (Bellet y Llop, 2004a). Conocer cuáles son sus características específicas y sus trayectorias recientes, determinar qué factores explican el dinamismo de algunas ciudades y el estancamiento o declive de otras, establecer tipologías entre la diversidad de modelos de ciudades en función de su especialización económica, de su perfil demográfico o de su posición en el territorio regional o nacional, así como establecer herramientas metodológicas adecuadas para el análisis de sus dinámicas internas y externas y para facilitar el análisis comparativo en territorios y contextos diferentes, han sido objetivos compartidos por un buen número de investigaciones realizadas desde los diversos campos científicos con interés por las dinámicas territoriales, entre los cuales, la Geografía desempeña un papel muy relevante (Brunet, 2000; Vilagrasa, 1999; Bellet y Llop, 2000, 2004a, 2004b; Ganau y Vilagrasa, 2003; Hall, 2000; Gaspar, 2000; Juaristi; 2000; Salom, 2000).

La atención prestada a estas cuestiones y el conocimiento progresivamente mayor de esta realidad y de su significado espacial, han contribuido a reforzar el interés por las áreas urbanas en general, y por las ciudades intermedias en particular, en los debates de política territorial. En el ámbito de la Unión Europea, los Estados miembros y la Comisión Europea, al tiempo que están enfatizando la importancia de la dimensión territorial de las políticas de cohesión económica y social, refuerzan también de forma sustancial la visibilidad del papel de las ciudades en las orientaciones estratégicas de desarrollo territorial.

\section{Las ciudades intermedias en las estrategias territoriales europeas}

Los principales documentos que contienen las estrategias territoriales de la Unión Europea, tales como, la Estrategia Territorial Europea (1999), el Programa de Estudio sobre Planificación Espacial Europea (2000), los Informes sobre la Cohesión Económica y Social, la Comunicación de la Comisión al Consejo y al Parlamento Europeo sobre Política de Cohesión y Ciudades (2006) o la Agenda Territorial de la Unión Europea (2007) coinciden en señalar que el logro de un espacio europeo más equilibrado exige la conformación un modelo de desarrollo policéntrico. Conservando la tradición de la diversidad urbana que es inherente a la historia de la construcción europea, se señala que ha de perseguirse la consolidación de un potente sistema urbano descentralizado, esto es, una compleja red de ciudades de dimensión grande, mediana y pequeña, como condición indispensable para conseguir el desarrollo equilibrado y sostenible de las ciudades y regiones y el aprovechamiento de su potencial económico. 
En este sentido, las transformaciones tecnológicas, económicas, sociales y políticas que influyen sobre el sistema urbano, sobre sus funciones y sobre su dinámica territorial, así como la necesidad de disponer de un potencial económico suficiente para asegurar la competitividad de las regiones europeas, exigen garantizar un acceso equivalente a las infraestructuras y al conocimiento, de manera que se favorezca la creación de oportunidades sobre la base de las fortalezas propias de las ciudades de diferente tamaño y se aminoren los desequilibrios territoriales en el potencial de innovación del espacio europeo.

Con el fin de orientar estos objetivos, los documentos de estrategia territorial establecen que las infraestructuras de transporte de alto nivel y grandes prestaciones integren adecuadamente las regiones periféricas con el núcleo central de UE - donde se concentran las funciones económicas y los servicios de elevado valor - y sean debidamente completadas con redes secundarias. De igual modo, las redes de telecomunicaciones -infoestructuras - deben complementar a las infraestructuras convencionales y reforzarse mutuamente para otorgar mayores ventajas al conjunto del sistema de ciudades y regiones.

No obstante, se advierte que la posibilidad de liberar el potencial innovador de los diversos territorios no está garantizada por la mera presencia de redes de transporte y telecomunicaciones sino que se requiere además otro tipo de acciones como son, la implementación de políticas dirigidas a la promoción de la educación y la formación de calidad, el acceso al conocimiento, la dotación de centros de investigación y enseñanza superior, así como de instituciones de transferencia de tecnología y difusión de las innovaciones que, en cualquier caso, deben adaptarse a las necesidades específicas de las estructuras económicas y sociales de cada región. De esta manera, las ciudades intermedias podrán y deberán desarrollar nuevas oportunidades a partir de sus propios recursos, diversificar y ampliar su base económica y generar atractivos para captar inversiones, lo que contribuirá a reforzar la posición competitiva de la UE en su conjunto en el escenario mundial.

Considerando pues la capacidad de las ciudades para la creación de entornos propicios a la innovación, para la formación de tejidos empresariales y para la generación de inversiones y empleos de calidad, se estima que su acción es determinante para lograr la cohesión económica, social y territorial. En consecuencia, la política de cohesión europea ha de apoyar cuantas iniciativas se orienten a reforzar su atractivo y sus ventajas competitivas, teniendo en cuenta que es a escala local donde pueden llevarse a la práctica acciones complejas que requieren el conocimiento de las características específicas del entorno.

Con todo, los documentos de política territorial ponen también de manifiesto las dificultades que deben enfrentar las ciudades pequeñas y medianas para responder con éxito a los desafíos económicos y sociales y para lograr una verdadera y eficiente movilización de sus recursos y activos territoriales. De ahí, que una de las orientaciones básicas se dirija a potenciar la configuración y consolidación de formas de cooperación en las que las ciudades deben actuar como partes de un modelo policéntrico a diversas escalas.

Por una parte, las múltiples interacciones funcionales de las ciudades con su entorno han de conducir a la creación de ámbitos de cooperación local con el fin propiciar una adecuada ordenación territorial e impulsar la competitividad del conjunto mediante el desarrollo de funciones complementarias y el aprovechamiento común de los recursos disponibles. La promoción de acciones integradas de desarrollo territorial de ámbito regional y sub-regional, se plantea pues como una opción estratégica que adquiere una especial relevancia en las regiones con menor densidad de población y económicamente más atrasadas, en las cuales, la creación de redes de ciudades favorece la combinación y la optimización de los potenciales urbanos. Por otra, en el marco de la cooperación transnacional y transfronteriza, las ciudades más alejadas entre sí pueden realizar proyectos conjuntos, intercambiar experiencias y encontrar soluciones a problemas comunes mediante la cooperación en re- 
des. Superar el reto de la competencia mundial exige crear oportunidades para el potencial económico innovador a partir de experiencias de cooperación que faciliten la promoción de agrupaciones, clusters de ciudades y redes urbanas, incluyendo el medio rural.

La configuración de este modelo de organización espacial en red exige al mismo tiempo la promoción de nuevas formas de asociación y gobernanza territorial, entendiendo por tal la interacción y el diálogo intensivo y continuo entre todos los agentes implicados en el desarrollo territorial, tanto en las regiones más densamente urbanizadas como en las articuladas por centros regionales y ciudades pequeñas. El establecimiento de formas de cooperación flexible entre los distintos niveles de las instituciones públicas - ciudades, regiones, Estados - debería facilitar la coordinación entre las políticas territoriales locales/regionales y las directrices europeas en la definición de sus políticas urbanas y de ordenación territorial. Esto implicaría superar la concepción del territorio como compartimentos vinculados a las estrictas competencias administrativas de ámbito local y regional para diseñar de manera coordinada la planificación espacial, enmarcando las estrategias de desarrollo en el contexto europeo. Es necesario para ello promover un «diálogo territorial» que permita a las autoridades regionales y locales participar en las negociaciones y en la toma de decisiones relativas a las políticas y acciones de la dimensión urbana, especialmente en el marco de las políticas de cohesión y de la gestión de los Fondos Estructurales.

En tal sentido, se subraya el reforzamiento del papel de las ciudades en las políticas territoriales ya que, de acuerdo con el reglamento de los Fondos Estructurales, los Estados miembros pueden delegar en las administraciones locales la gestión de los recursos destinados a determinadas acciones urbanas incluidas en los programas operativos, al tiempo que las ciudades pueden recurrir a la asistencia técnica de los Fondos Estructurales para gestionar todos los aspectos del desarrollo urbano (Agenda Territorial de la Unión Europea, 2007).

Con el fin de favorecer que las ciudades asuman un papel más activo en la integración de sus estrategias de desarrollo en el contexto europeo, desde las instancias comunitarias se anima a la ampliación y consolidación de los programas y observatorios de políticas urbanas orientados a facilitar el intercambio de conocimientos, experiencias y buenas prácticas urbanas así como a analizar en profundidad los efectos territoriales de las políticas de cohesión y a establecer alianzas estables de cooperación entre ciudades y redes urbanas.

\section{El impulso de las redes urbanas de cooperación}

La relevancia que las ciudades están adquiriendo en el marco de las políticas y estrategias territoriales comunitarias ha llevado consigo el desarrollo de un amplio abanico de iniciativas orientadas a favorecer un conocimiento más profundo y riguroso de la realidad urbana europea. Entre ellas cabe destacar, el desarrollo de programas de apoyo a la creación redes de ciudades y observatorios en red, la realización de estudios y auditorías urbanas a escala europea o el diseño de herramientas apropiadas para valorar los impactos territoriales de las políticas de cohesión.

Aunque las primeras iniciativas comunitarias en el ámbito de las redes urbanas se remontan a finales de los años ochenta, es en 1991 cuando se inicia el Programa Recite con el fin de promover la cooperación entre ciudades y regiones para la realización de proyectos comunes. En sus objetivos figura la promoción económica de las regiones desfavorecidas, la generación de economías de escala mediante la cooperación, y la implementación de las políticas regionales comunitarias (Boix, 2003 y 2006). Entre otras redes participantes en este programa destaca Eurocities, a la que actualmente pertenecen más de 130 grandes ciudades europeas. Esta red constituye una plataforma para compartir conocimientos e 
ideas, intercambiar experiencias, analizar problemas comunes y desarrollar soluciones innovadoras. Además representa a sus miembros ante las instituciones europeas en los aspectos referidos a la legislación comunitaria, las políticas y los programas con impacto en las ciudades. También aborda proyectos transfronterizos en relación con el desarrollo económico y la cohesión social, la provisión de servicios públicos, la sostenibilidad ambiental, el transporte y la movilidad urbana, la educación y la cultura, los asuntos sociales y el empleo, la sociedad de la información y el conocimiento, la gobernanza territorial y la cooperación internacional (Eurocities, 2007).

Por su parte, la Dirección General de Política Regional puso en marcha en 1999 el Proyecto Piloto de Auditoría Urbana mediante la actuación conjunta de las agencias estadísticas nacionales y las autoridades municipales coordinadas por Eurostat. Tras la evaluación de este proyecto experimental, en 2003 y 2004 se realizó la primera Auditoría Urbana Europea a gran escala que tiene continuidad cada tres años con la compilación de indicadores y variables de más de 250 ciudades europeas, de manera que puedan realizarse comparaciones objetivas entre las ciudades en los ámbitos de la demografía, las condiciones sociales, los aspectos económicos, la educación, la cultura, la participación ciudadana, el transporte y el medio ambiente (Urban Audit). Sobre esta base estadística, en 2007 se elaboró el informe titulado Estado de las Ciudades Europeas (ECOTEC, NordRegio y Eurofutures) que proporciona un análisis exhaustivo del cambio demográfico, la competitividad urbana, las condiciones de vida y el poder administrativo de las ciudades.

Entre las principales conclusiones del informe (Comisión Europea, 2007), se subraya el papel de las ciudades como motores del crecimiento económico y como principales generadores de conocimiento e innovación y se advierte que, aunque las ciudades de mayores dimensiones contribuyen en mayor medida a la buena marcha de la economía, las pequeñas ciudades europeas muestran tasas de crecimiento económico que coinciden con la media. Al mismo tiempo, la acelerada transformación de las economías urbanas en economías de servicios hace que en los mercados laborales urbanos este sector sea la principal fuente de empleo. El estudio pone de manifiesto, asimismo, las disparidades en los indicadores de competitividad urbana a escala europea - las ciudades que mejores resultados arrojan se localizan en el norte y centro de Europa - pero también en las escalas nacionales - Italia, Reino Unido y Bélgica cuentan con ciudades que figuran entre las mejor y peor situadas - indicándose que los niveles de competitividad no guardan relación con el tamaño de la ciudad, ya que tanto las ciudades grandes como las pequeñas pueden obtener resultados muy positivos.

La dimensión, la estructura, los resultados económicos y los factores impulsores de competitividad, son los criterios considerados en el informe para el establecimiento de una tipología de ciudades que se jerarquiza en tres niveles. Los núcleos internacionales de Europa integrados por los núcleos de conocimiento - por encima de la jerarquía nacional urbana y en la vanguardia de la industria, los negocios y los servicios financieros - ; las capitales establecidas - con una base económica diversificada y fuerte concentración de riqueza-; y las capitales «reinventadas» - motores de actividad económica para los nuevos Estados miembros-. En un segundo nivel, se sitúan los polos especializados que pueden desempeñar un papel destacado en determinados aspectos de la economía urbana: núcleos nacionales de servicios, polos de transformación, centros industriales modernos, centros de investigación y educación superior, centros de visitantes y puertas de entrada. Finalmente, en un tercer escalón se distinguen los polos regionales, considerados como los pilares de las economías regionales europeas: las ciudades desindustrializadas en transformación, los centros regionales de mercado (servicios personales, negocios, financieros, comerciales...), los centros regionales de servicios públicos (administrativos, sanitarios, educativos...) y las ciudades satélite (con un papel específico en las aglomeraciones urbanas). 
Con independencia de que determinadas ciudades puedan figurar en más de una categoría, esta propuesta se considera una herramienta útil para interpretar las dinámicas urbanas contrastadas y las diferencias entre las distintas ciudades en función de la forma en la que se utilizan y se combinan los factores impulsores de la competitividad, esto es, la innovación, el talento, el espíritu empresarial y la conectividad. En consecuencia, lo que determinaría el éxito económico de las ciudades es la forma de utilizar los recursos clave que se encuentran a su disposición, por lo que el conocimiento de estas realidades diversas y el intercambio de experiencias pueden ayudar a abordar más fácilmente la combinación de las actuaciones y las políticas urbanas más adecuadas para los distintos tipos de ciudades. En este sentido, otra de las aportaciones interesantes del estudio se refiere precisamente al poder de las administraciones locales - mediante el «índice del poder relativo» de los gobiernos municipales que está basado en el tamaño, la estructura administrativa, los ingresos fiscales y los gastos municipales - que determina la capacidad de las ciudades para formular y aplicar estrategias que les permitan abordar los desafíos económicos, sociales y territoriales.

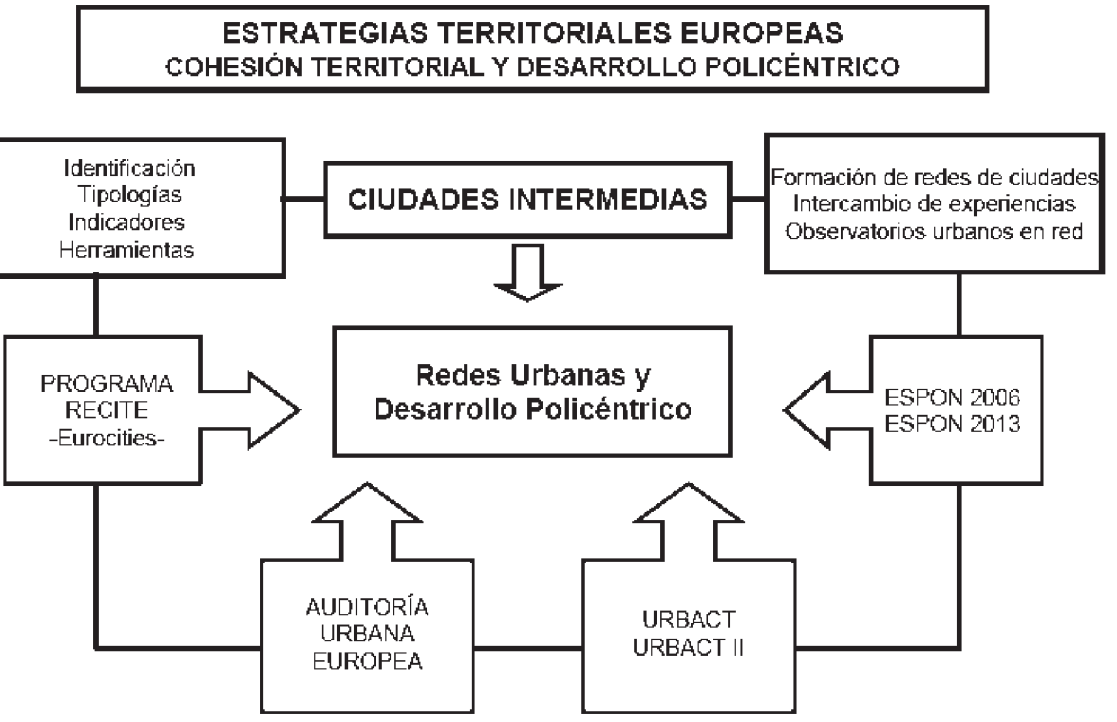

FIgURA I. Redes urbanas y desarrollo policéntrico. Elaboración propia.

Paralelamente al desarrollo de esta auditoría urbana, en el marco de la Iniciativa Comunitaria Urban II la Comisión aprobó el programa UrbAct (2002-2006) cuyos objetivos se centran en el intercambio de buenas prácticas entre las ciudades participantes en Urban, la difusión de las experiencias adquiridas y la mejora de la capacidad de acción de las ciudades. Para ello el programa propone como ejes prioritarios la creación de redes temáticas para difundir los conocimientos, la realización de informes y el asesoramiento de expertos. Actualmente este programa tiene continuidad en UrbAct II, el cual, sobre la experiencia acumulada en el anterior, continúa apoyando este tipo de actuaciones habiendo ampliado el abanico temático y el número de ciudades elegibles (los participantes son las ciudades de los 27 Estados miembros, las regiones, las universidades y los centros de investigación). 
Amparado por la Iniciativa Comunitaria Interreg III (2002-2006) se creó también el Observatorio en Red para la Planificación Espacial Europea - ESPON en sus siglas inglesas - . El programa ESPON 2006 se constituye como una red de observación y de cooperación entre institutos nacionales de ordenación territorial que se ocupa del análisis y de las tendencias del desarrollo territorial mediante la elaboración de proyectos y estudios articulados en tres ejes prioritarios: los dirigidos a promover la aplicación de la Estrategia Territorial Europea, los que se centran en el análisis de los efectos territoriales de las políticas comunitarias y los que desarrollan las herramientas básicas de análisis (metodologías, bases de datos e indicadores de desarrollo territorial). Desde su creación, ESPON financia y monitoriza proyectos de investigación aplicados que cubren un amplio abanico de temáticas espaciales, incluyendo diversos estudios referidos al significado de las áreas urbanas en el desarrollo policéntrico, el sistema urbano europeo, las funciones urbanas o el papel de las ciudades pequeñas y medianas en el desarrollo regional. En relación con este último aspecto, el informe elaborado en 2006 pone de relieve cómo los estudios urbanos se han centrado fundamentalmente en las grandes ciudades y metrópolis, y subraya el hecho de que, pese a que las políticas comunitarias otorgan a las pequeñas y medianas ciudades - SMESTO - un significado cada vez más relevante, los análisis sistemáticos de este tipo de ciudades son escasos y la información estadística disponible a este nivel es muy limitada y no permite establecer análisis comparativos.

Para avanzar en la superación de estas carencias, el informe aporta una propuesta de conceptualización de las pequeñas y medianas ciudades, tanto desde el punto de vista morfológico como funcional y administrativo, y establece una tipología basada en tres criterios: su posición espacial (aglomeradas, en red, aisladas...), su dinamismo socio-económico (en crecimiento, en declive, en reestructuración, con desarrollo potencial...) y su organización político-administrativa. El informe pretende ser un estudio preparatorio para orientar la investigación empírica de las pequeñas y medianas ciudades europeas que se realizará en el programa ESPON 2013, iniciado en enero de 2008.

Las propuestas del nuevo programa se articulan sobre la realización de estudios y proyectos de investigación coordinados (potencial de las ciudades y aglomeraciones urbanas en la competitividad europea, oportunidades de desarrollo para las áreas rurales, tendencias demográficas y flujos migratorios en las ciudades y regiones europeas, efectos del cambio climático en las regiones y economías locales, efectos de los precios de la energía en la competitividad regional, evaluación del impacto territorial de las políticas públicas), la creación de bases de datos comunes y la formación de equipos de expertos en materia territorial.

En suma, los avances en el conocimiento de las dinámicas territoriales europeas, la valoración de los impactos de los programas europeos en las ciudades y el análisis de las políticas y estrategias urbanas, ponen de relieve la importancia y la naturaleza específica de los diversos niveles de intervención pública en el territorio y la multiplicidad de las interacciones que se generan entre ellos. Centrando la atención sobre los aspectos referidos al nivel local de intervención, la experiencia acumulada durante los últimos años refleja de qué manera han evolucionado las políticas urbanas, ampliando el abanico de objetivos y actuaciones y diseñando nuevos instrumentos que ponen el acento en el reforzamiento de los factores de competitividad urbana.

\section{De las políticas urbanas de promoción y ordenación a las políticas de planificación estratégica}

Tradicionalmente las competencias de las administraciones locales en la promoción económica de las ciudades se han limitado a la mera regulación de la actividad de las 
empresas, al establecimiento de los criterios de localización de las actividades económicas en la ciudad, mediante el planeamiento urbanístico, y a la ordenación de suelo planificado para albergar la actividad empresarial. Si bien en la actualidad algunas ciudades siguen circunscribiendo su capacidad operativa a este tipo de intervenciones, es cada vez más habitual que los poderes locales asuman una concepción mucho más amplia de su capacidad de intervención en favor del desarrollo de una política urbana de carácter integrador que, adoptando una perspectiva y un enfoque estratégico, reorienta las prioridades de la intervención urbana hacia el crecimiento y el reforzamiento de la competitividad (Brugué y Gomà, 1998; Rodríguez, Moulaert y Swingedouw, 2001; Vilagrasa, 2003; Méndez, 2006; Ezquiaga, 2006; Benito, 2004).

El aumento de la complejidad de las ciudades, supone también un incremento en la dificultad de la gestión de los problemas y actividades administrativas que deben acometer para ofrecer un servicio eficaz a los ciudadanos. En este sentido, los instrumentos de gestión urbana tradicionales no son suficientes para afrontar las interrelaciones que se establecen entre diferentes problemas o disfunciones urbanas, por lo que es preciso acudir a otras fórmulas de planificación más compleja e integradora. Podemos mostrar algunas de estas situaciones tratando de agrupar en seis apartados los problemas actuales a los que deben hacer frente las ciudades. El primero de ellos viene definido por la creciente complejidad e interrelación entre los problemas urbanos tradicionales. Así, el incremento del tráfico, la escasez y carestía de la vivienda, la seguridad ciudadana, la marginación social, la llegada de nuevos contingentes poblacionales, etc., han sido una constante entre las preocupaciones de los gestores urbanos. Pero a ellos se añaden otros como el debilitamiento de la base económica, la pérdida de atractivo de la ciudad para las nuevas actividades empresariales o el deterioro del medio ambiente, para los que las ciudades no estaban tradicionalmente preparadas, o a los que daban soluciones políticas otras administraciones territoriales de rango superior. En relación con lo anterior, aparece un segundo grupo de problemas derivados de una mayor incertidumbre del entorno geopolítico, económico y tecnológico, como consecuencia de la globalización de los mercados, de la división internacional del trabajo, de la implantación del Mercado Único o de la emergencia de nuevas economías.

El tercer apartado vendría definido por los problemas derivados del incremento de la competencia entre ciudades, de tal modo que la búsqueda de atractivos para captar empresas, inversiones, visitantes, infraestructuras, equipamientos y ayudas regionales o estatales, se convierten en una carrera de obstáculos en la que rivalizan numerosas ciudades o áreas urbanas, para tratar de conseguir esos beneficios en detrimento de sus competidoras. Pero, para ello, deben llevarse a cabo propuestas de planificación a medio y largo plazo sobre aspectos que sobrepasan las competencias de los propios ayuntamientos. El cuarto desafío al que se enfrentan las ciudades está relacionado con el protagonismo que ejercen $-\mathrm{y}$ desean ejercer - en la recuperación económica de sus regiones circundantes o ámbitos de influencia. En este sentido, las ciudades juegan un importante papel como impulsoras del desarrollo económico, como centros de innovación social, cultural y técnica, y como núcleos de integración comunitaria. Para afrontar estos desafíos, las ciudades deben liderar un proceso de mayor implicación participativa de los diferentes agentes sociales. Y éste aspecto se convierte en el quinto apartado de su reciente problemática, por cuanto es preciso que la administración pública, los empresarios, los sindicatos, los movimientos ciudadanos, las universidades y, en definitiva, la sociedad civil organizada, tengan una mayor participación e información sobre las decisiones públicas que afectan al futuro de la ciudad. Al mismo tiempo, otra exigencia en esta misma línea procede de los contribuyentes que demandan mayor coordinación institucional y eficacia en las acciones, con el fin de evitar duplicidades de acciones y actividades contrapuestas. 
Por todo ello, y éste podría ser el sexto grupo de problemas, las ciudades ponen de manifiesto las limitaciones de los instrumentos tradicionales de planificación, al adolecer de un exceso de rigidez, ser sectoriales y especializados, prestar mayor atención al producto u objetivo perseguido que al proceso y los medios para conseguirlo, ser poco participativos y apenas involucrar a la iniciativa privada. Se plantea, así, la necesidad de desarrollar nuevas formas de gestión con mayor capacidad operativa, al tiempo que se pretende ofrecer una visión integradora de los problemas poniendo en relación las causas y consecuencias que cualquier actuación pública puede tener sobre otros aspectos de la realidad urbana. Así, de los avances en el proceso de renovación de las políticas de desarrollo urbano, son fiel reflejo los planteamientos que subyacen en los planes estratégicos que comienzan a diseñarse en las grandes metrópolis durante la última década del pasado siglo, y que han alcanzado desde entonces una gran difusión en todo tipo de ciudades con independencia de su tamaño, debido a la creciente complejidad a la que se enfrenta la gestión y la planificación de las áreas urbanas.

En esencia, el cambio fundamental de la planificación estratégica respecto a los principios de la planificación tradicional consiste en su identificación como un proceso de reflexión en el que participan el conjunto de agentes sociales públicos y privados con el fin de definir el proyecto de ciudad y diseñar las estrategias y los proyectos adecuados para la consecución de los objetivos previstos, un instrumento para formular un proyecto global de ciudad. La idea fundamental es que el futuro de la ciudad no es un problema exclusivo de la Administración, sino que afecta a toda la población y a los agentes sociales y económicos. El plan define las líneas prioritarias a partir de las cuales se deberán realizar los planes de actuación de los agentes urbanos públicos y privados y, en particular, el de la administración local, y contribuye a difundir el pensamiento estratégico como marco de referencia para la toma de decisiones (Forn, 1991: 292)

Las principales ventajas de la planificación estratégica se derivan de su capacidad para ofrecer una visión global e intersectorial del sistema urbano o regional; identificar tendencias y descubrir oportunidades; formular objetivos prioritarios; concentrar recursos limitados en temas críticos; generar consenso y compromiso comunitario para la acción; fortalecer el tejido social; promover la coordinación entre instituciones; implicar a la iniciativa privada; y otorgar alta visibilidad política y social (Fernández Güell, 1991: 289). Estas son las principales diferencias que subyacen entre ambos modelos de planificación. Como puede observarse en el cuadro 1, la planificación estratégica trata de suscitar un mayor consenso entre los diferentes agentes, afronta los problemas desde una perspectiva más global e integradora, propone soluciones a medio y largo plazo, estimula la participación de todos los agentes implicados, permite la revisión de las actuaciones y de los progresos logrados a través de prácticas de gobernanza local, y la realización de diagnósticos globales de problemas que aparentemente pueden parecer locales o sectoriales. Su elaboración exige pues un diagnóstico previo para evaluar la situación de la ciudad, sus debilidades y sus potencialidades; requiere aunar intereses diversos y alcanzar acuerdos que permitan plantear una visión estratégica de la ciudad a través de la definición de las ideas clave sobre las cuales se establecerán las líneas maestras de trabajo que posteriormente han de cristalizar en planes y proyectos concretos. Como herramienta de planificación a largo plazo, el plan estratégico lleva consigo un proceso posterior de seguimiento y evaluación de las actuaciones previstas, de su grado de cumplimiento y de su impacto en la ciudad (Pascual y Forn, 1999; Gómez, 2003; Mella, 1999). Este planteamiento integrador para definir los problemas y aportar soluciones, junto con la pretensión de actuar más allá de los límites administrativos sobre los que se tiene jurisdicción, son los elementos más singulares que la diferencian de la planificación tradicional o normativa. 


\section{Cuadro 1 \\ DIFERENCIAS GENÉRICAS ENTRE PLANIFICACIÓN NORMATIVA Y PLANIFICACIÓN ESTRATÉGICA}

\begin{tabular}{|l|l|}
\hline Planificación Normativa & Planificación Estratégica \\
\hline - Exclusión del conflicto & - Consenso para conciliar conflictos \\
- Formula decisiones & - Efectúa dirección y gestión \\
- Imposición de utopía en realidad & - Ajusta objetivos a la realidad \\
- Rigidez para cambiar/adaptarse y en & - Flexibilidad para adaptarse a cambios, \\
temas y métodos & temas y métodos \\
\hline - Fracaso ante la crisis urbana & - Adaptación de medios a los diagnósticos \\
- Simplificación de diagnósticos & - Diagnósticos de tipo «prospectivo» \\
- No se retroalimenta de sus efectos & (sobre competencias en mercados eco- \\
- Falta de relación inter-administracio- & nómicos) \\
nes & - Objetivos a largo plazo \\
& - Visión global e integrada: Administra- \\
& ciones, territorio, entornos económicos \\
\hline - Ordena espacio urbano & - Planifica desarrollo no solo urbano \\
- Determina y localiza usos del suelo & - Proyectos no solo territorializables \\
\hline Administración responsable & - Consenso entre agentes públicos y pri- \\
- Participación a posteriori & vados \\
- Normas para regular acción privada & - Participación social en todas las fases \\
futura & - Compromisos para actuar en corto \\
& plazo \\
\hline $\begin{array}{l}\text { Tomado de Martínez López, M. (1999). Fuentes: Ander-Egg (1991), Vázquez (1993), Enguita } \\
\text { (1993), Borja y Castells (1997). }\end{array}$ \\
\hline
\end{tabular}

Otra de las diferencias sustanciales entre la planificación estratégica y las propuestas de promoción y ordenación tradicionales, se encuentra en el enfoque metodológico que supone una mayor complejidad en su elaboración y ejecución, pero que aporta un conocimiento más exhaustivo y profundo de la realidad territorial sobre la que deseamos intervenir. Las principales etapas o fases que debe contener la redacción de un Plan Estratégico serían las siguientes (Fernández Güell, 1991 y 2006): a) Organización del plan, que implica la adaptación de la metodología a las exigencias concretas del proyecto, el diseño del modelo organizativo del plan, la participación de los agentes sociales, y la redacción de un programa de comunicación y difusión del plan. b) Exploración del medio e identificación de los temas críticos, con el fin de incorporar una visión comprensiva de la comunidad, que contemple tanto los acontecimientos pasados, como las condiciones actuales y las previsiones futuras, al mismo tiempo que debe identificar con claridad los asuntos más sensibles a fin de abordarse con prioridad. c) Análisis DAFO, con una vertiente de análisis externo que pueda anticipar las tendencias sociales, económicas, tecnológicas y políticas (oportunidades y amenazas), al tiempo que valore los puntos fuertes y débiles de la comunidad a partir de las tendencias del entorno. d) A partir del análisis anterior, deben fijarse metas y objetivos que sean realistas y alcanzables (realizables), al tiempo que los objetivos puedan ser cuantificables. e) Desarrollo de estrategias dirigidas al cumplimiento de los objetivos propuestos. Éste es el primero de los apartados operativos y, por tanto, las estrategias deben ser consensuadas 
y priorizadas - además de acordes con los presupuestos-recursos asignados - , a fin de que puedan garantizar el éxito en su ejecución. f) Todo lo anterior debe llevarse a cabo por medio de un plan de acción, en el que se cuantifiquen los recursos necesarios y se asignen responsabilidades concretas para implantar las estrategias seleccionadas. g) Finalmente, el último paso es la implantación del plan de acción. Esta es sin duda la etapa más importante, porque depende de la movilización-compromiso de los agentes sociales que han participado en la elaboración del plan para que deje de ser una propuesta de actuación y pase a ser una realidad. Es el punto en el que se pasa de redactar documentos propositivos a convertirlos en herramientas ejecutivas de planificación.

La planificación estratégica urbana ha sido objeto de no pocas valoraciones críticas que cuestionan tanto las bases ideológicas sobre las que se asienta, como su validez como instrumento de planificación y los limitados resultados que en ocasiones arroja (Enguita, 1993; Precedo, 1993; Martínez, 1999; Areilza y Urrutia, 2000). No obstante, en la trayectoria evolutiva de las políticas urbanas no cabe duda de que esta forma de planificación constituye un hito relevante al ofrecer una visión más coherente e integradora, que supera las actuaciones sectoriales aisladas y la frecuente disociación entre las intervenciones urbanísticas y las dirigidas a la promoción económica de la ciudad (Méndez, 1995).

Así, el desarrollo de un plan estratégico implica el establecimiento de diferentes Áreas Estratégicas de Acción por medio de las cuales se puede llevar a cabo esa gestión integrada a la que venimos haciendo referencia (Pascual Esteve, 2001: 32). La primera de éstas Áreas Estratégicas vendría definida por todos los aspectos territoriales que se encuentran interrelacionados. De entre todos los posibles, podemos señalar la necesidad de concebir un espacio bien conectado internamente, con una alta accesibilidad entre los distintos ámbitos urbanos - residenciales, productivos, comerciales, asistenciales, etc.- - , pero también con los espacios exteriores, ya sean los inmediatamente circundantes como aquellos otros más alejados que forman parte de la misma red de ciudades, buscando una mayor integración territorial. Otro caso sería la necesidad de recuperar y revitalizar los centros históricos al tiempo que se van creando e integrando en la ciudad las nuevas áreas urbanas residenciales, comerciales, logísticas o industriales. La aparición de estos nuevos espacios y de las actividades a ellas asociadas generan nuevas áreas de centralidad que es preciso integrar en el conjunto urbano y a las que hay que dotar de accesibilidad y comunicación con el resto de la ciudad y con la red urbana regional. Al mismo tiempo, todas estas modificaciones urbanas deben llevar implícitas acciones correctoras del impacto medioambiental, así como actuaciones encaminadas a la recuperación y puesta en valor de los espacios naturales existentes.

La segunda de las áreas estratégicas estaría relacionada con todos los aspectos de cohesión social, tales como la reducción de las desigualdades sociales, el impulso a programas que ofrezcan nuevas oportunidades sociales y económicas a las clases menos favorecidas, la promoción del asociacionismo y la participación, la adecuación de la red de equipamientos y servicios sociales a las necesidades de una sociedad cada vez más compleja, diversa y amplia, y finalmente, el fomento de la educación media y superior adaptada a la realidad socioeconómica y a la demanda de profesionales cualificados.

En un tercer apartado, debemos considerar todos los aspectos relacionados con el gobierno de la ciudad que debe estar orientado en varias direcciones. Debe contemplarse el establecimiento de mecanismos de cooperación social y participación ciudadana, en los términos que recogen las actuales propuestas sobre gobernanza, que permitan colaborar en la gestión de las ciudades, en su toma de decisiones pero también en el control de la eficacia, calidad y buenas prácticas que se deben exigir a las administraciones públicas. Debe primarse la adecuación de las actuaciones municipales a las necesidades reales de 
la población, al tiempo que debe exigirse una mejora en la gestión municipal. Paralelo a lo anterior, otra estrategia de gobierno de la ciudad debe encaminarse a la mejora de la colaboración interinstitucional, que ponga en perfecta relación las actuaciones de las diferentes administraciones del Estado, y de cada uno de los diferentes departamentos de éstas entre sí - entre ministerios, consejerías, concejalías, direcciones generales, secretarías, etc. - Esta perfecta coordinación podrá permitir, al mismo tiempo, la creación o formalización de relaciones con otros territorios, con el fin de crear redes de innovación o de cooperación. La transferencia de conocimientos y prácticas entre distintas ciudades de un mismo entorno territorial, redundará en el aprovechamiento de unas sinergias que permitirán aprovechar mejor los recursos disponibles y avanzar más rápidamente para la consecución de determinados objetivos.

Por último, la cuarta de las áreas estratégicas de acción está relacionada con las actividades económicas, ya que éstas desempeñan un doble papel sobre la planificación de cualquier territorio. Por un lado, porque son las que demandan unas determinadas condiciones en los planos normativo y funcional para que se den las condiciones óptimas que requiere el capital para su desarrollo financiero y, por extensión, para su expansión productiva, laboral y comercial. Por otro, porque se trata de un sector muy sensible a determinadas coyunturas y ciclos, al tiempo que busca los territorios mejor dotados y desarrollados logísticamente para implantarse. Por estas razones, las líneas de actuación estratégica deben ir dirigidas a promover determinados sectores económicos o productivos, especialmente los relacionados con el desarrollo endógeno del propio territorio, al tiempo que debe mejorarse la eficacia de los procesos productivos y la competitividad de los servicios. Además de procurar la mejora de las condiciones para las empresas y actividades ya radicadas, las actuaciones propuestas deben estimular la creación de nuevas empresas en el territorio que conlleve la implantación de diferentes actividades, en la búsqueda constante de ampliar y diversificar especializaciones productivas, al tiempo que contribuirá a la creación de nuevas áreas de centralidad.

En consonancia con estos planteamientos, la nueva generación de planes estratégicos diseñados en los últimos años en las ciudades medias parten de una visión integradora de las políticas urbanas, abordan con un enfoque globalizador un abanico de objetivos muy amplio y diversificado y habitualmente cuentan con instrumentos flexibles de gestión capaces de adaptarse con rapidez a los continuos cambios económicos, tecnológicos, sociales y territoriales que establecen nuevos retos y desafíos al gobierno de la ciudad.

\section{Estrategias urbanas para la promoción de la innovación y la generación de ventajas competitivas}

Como ya se ha señalado, el progreso de las ciudades intermedias depende de la capacidad institucional local para aprovechar los recursos endógenos, de la voluntad para desarrollar un proyecto de ciudad, de su grado de cohesión y cooperación de los agentes sociales, de las estrategias de especialización competitiva, así como de la capacidad para adoptar y generar innovaciones desarrollando sus propias estrategias (Bellet y Llop, 2004a). Así, las nuevas políticas urbanas orientadas a la creación de entornos innovadores, capaces de activar sus ventajas competitivas, parten de una concepción de la innovación que supera su carácter meramente físico, técnico y lineal para entenderse como un proceso colectivo de aprendizaje, apoyado en el entramado de agentes sociales, redes de instituciones y entidades públicas y privadas que interactúan entre sí generando un proceso de aprendizaje en el que se crea, se intercambia y se produce conocimiento (Moulaert y Lamboy, 1998; Aguado, 2005; Arboníes, 2005). En consecuencia, el proceso innovador estaría determinado por un contexto concreto, por un ámbito territorial con características y especificidades propias cuyos elementos - agen- 
tes, recursos, interacciones...- se identifican con ese espacio y sólo pueden interpretarse en relación con él (Méndez, 2001 y 2002; González, 2006). Así, algunas sociedades/territorios son capaces de crear entornos favorables a la innovación a través de la movilización de sus recursos territoriales específicos, tanto materiales como inmateriales, entre otros, la cultura técnica y empresarial, los recursos humanos (cualificaciones, formas de trabajo, habilidades...), la capacidad de cooperación entre los agentes, empresas e instituciones, el tejido empresarial, el compromiso y la implicación de las administraciones regionales y locales, la cohesión social, los valores sociales y culturales, etc. Por tanto, en la generación de ventajas competitivas urbanas cada vez tienen menos importancia los recursos genéricos, fácilmente reproducibles en lugares diversos y basados en la obtención de bajos costes, para otorgar mayor preponderancia a la movilización de los recursos territoriales específicos y actores locales con el fin de incrementar la capacidad de atracción de la ciudad (Rodríguez, Moulaert y Swingedouw, 2001; Méndez, Michelini y Romeiro, 2006).

Desde este enfoque, cobra mayor protagonismo el ámbito local de intervención en la definición de estrategias de promoción de la innovación ya que es en este nivel donde es posible adoptar una perspectiva endógena para realizar un diagnóstico adecuado de las potencialidades y debilidades del entorno, detectar, movilizar y potenciar los factores de desarrollo territorial, y facilitar la integración horizontal de las políticas urbanas con una perspectiva sistémica que reconoce la existencia de múltiples elementos interactuantes (Castillo y Díez, 2006; Keating, 2005).

Con el fin de sistematizar las líneas estratégicas de intervención pública en las ciudades intermedias y la tipología de actuaciones específicamente encaminadas a la promoción de la innovación como elemento clave de la competitividad urbana, hemos tomado como referencia los planes estratégicos de diez ciudades medias españolas - Vigo, Gijón, Vitoria, San Sebastián, Burgos, Valladolid, Segovia, Córdoba, Murcia y Elche - centrando fundamentalmente la atención en aquellas acciones que aparecen en dichos planes con enunciados coincidentes o que al menos presentan cierta similitud y que, en tal sentido, pueden ser interpretadas como reflejo de una tendencia general en el momento actual.

\subsection{La promoción del conocimiento como recurso estratégico y la cualificación de los recursos humanos}

En términos generales, el denominador común que justifica la puesta en marcha del proceso de planificación estratégica se vincula a los profundos cambios económicos, tecnológicos y empresariales derivados del proceso de globalización económica y del desarrollo de la sociedad de la información y del conocimiento, de manera que la participación activa en este proceso constituye uno de los retos esenciales de las ciudades intermedias que pretendan ser competitivas y avanzadas.

Eso explica que una de las líneas estratégicas más importantes en el diseño de las políticas urbanas de innovación se centre en la potenciación de la capacidad de generación de conocimiento y creatividad, como factores intangibles para lo que se requiere activar las potencialidades de todos los agentes implicados en los procesos de innovación y, a la vez, favorecer las relaciones de interacción entre los mismos. En tal sentido, los documentos de planificación estratégica plantean la importancia de crear un entorno adecuado para el desarrollo de actividades empresariales innovadoras e intensivas en conocimiento, e impulsar el fomento de la innovación, no sólo como esfuerzo de modernización de infraestructuras y equipos sino, sobre todo, como estímulo a la creación o a la consolidación de una cultura empresarial emprendedora y como potenciación de la capacidad de adaptación al cambio tecnológico y a la innovación. 
Es necesario para ello, estimular de forma activa la colaboración entre el entramado empresarial, los centros tecnológicos y los centros de investigación, y potenciar las entidades de transferencia tecnológica con el fin de dinamizar los flujos de conocimiento desde los focos existentes a las organizaciones que forman el tejido productivo y social de la ciudad, lo que contribuirá a aumentar la competitividad de la actividad económica. En este tejido de actores que lideran los procesos de innovación, las universidades aparecen como uno de los principales agentes de dinamización urbana, tanto en su calidad de generadores de conocimiento como en su calidad de centros de formación y cualificación de los recursos humanos.

Así, los planes estratégicos analizados coinciden en señalar que la oferta educativa universitaria ha de potenciar la configuración de sistemas formativos de calidad para la formación de profesionales de alta cualificación, capaces de poner en marcha nuevos proyectos y actividades innovadoras generadoras de riqueza. En esta misma línea, se enfatiza la conveniencia de implicar a las empresas en el diseño de los planes formativos, de adecuar los recursos educativos a los avances tecnológicos, así como de identificar los principales grupos de investigación, fomentar los contactos con los equipos de investigación más avanzados y promover su participación en redes de conocimiento.

\subsection{El apoyo al tejido empresarial, la formación/consolidación de cluster innovadores y la diversificación de la economía local}

El logro de una mayor capacitación profesional de los recursos humanos ha de ir acompañado de acciones encaminadas a facilitar el anclaje del capital humano e intelectual y a evitar su pérdida en favor de los grandes centros urbanos y económicos. Esto se concreta en propuestas como, la atracción de empresas y actividades económicas intensivas en conocimiento y el fomento al desarrollo de iniciativas empresariales locales innovadoras. En este ámbito, se considera que el papel de las administraciones públicas es fundamental tratando de apoyar el desarrollo de los proyectos empresariales en sus etapas iniciales y facilitando el acceso al capital-semilla y capital-riesgo, al apoyo técnico especializado y a los servicios avanzados. Con este objetivo, se está avanzando en la creación de centros de dinamización empresarial, ventanillas únicas, agencias de desarrollo empresarial y entidades similares, aunque con denominaciones muy diversas, en las que la participación de las administraciones locales se acompaña frecuentemente de otros agentes públicos y privados (las agencias regionales, las asociaciones empresariales, las organizaciones profesionales, las cámaras de comercio, las universidades, etc.).

En las ciudades intermedias, se trata también de vincular la atracción de empresas y la promoción de nuevas iniciativas locales con la consolidación del tejido industrial existente, especialmente cuando está configurado por pequeñas y medianas empresas. En este sentido, se enfatiza las dificultades a las que con frecuencia se enfrentan estas empresas para insertarse en entornos crecientemente globalizados y altamente competitivos, y se plantea la necesidad de estimular su participación en los procesos de investigación y desarrollo innovador, tanto en la mejora de los procesos productivos o de los servicios que suministran como en materia de gestión empresarial, incorporación de tecnologías de la información y la comunicación y búsqueda de nuevos mercados nacionales e internacionales.

En esta misma línea de apoyo al tejido empresarial, uno de los ejes prioritarios comunes a los planes estratégicos que hemos tomado como referencia se orienta a la potenciación de cluster sectoriales, entendidos como sistemas basados en las relaciones de interacción propiciadas por la proximidad territorial de las empresas industriales, los servicios especializados, las instituciones, las organizaciones de apoyo a la innovación y 
los centros de investigación/formación, en los que su funcionamiento conjunto favorece el acceso a información especializada, la cooperación en proyectos de innovación y la transmisión y difusión de los conocimientos y el aprendizaje entre los diversos componentes del sistema.

Además de apoyar estas líneas de acción y promover los procesos de innovación en las actividades productivas y de servicios ya existentes, los planes coinciden en indicar la conveniencia de estimular el crecimiento de sectores emergentes y nuevos yacimientos de empleo. Aunque, como es lógico, las referencias sectoriales concretas dependen en gran medida de las potencialidades y recursos de cada ciudad, los sectores emergentes suelen estar vinculados a polos de innovación relacionados con las biociencias - salud, biotecnologías, sector biosanitario - , la tecnología, la consultoría y el asesoramiento medioambiental, las energías renovables, el sector agroalimentario y la nutrición, el sector audiovisual, la producción cultural y la gestión de los recursos patrimoniales, los servicios turísticos avanzados, los servicios de ocio y tiempo libre, las actividades logísticas y los servicios empresariales especializados.

\subsection{El acondicionamiento de infraestructuras tecnológicas y empresariales}

Para dar soporte a estas iniciativas, los planes estratégicos promovidos por las administraciones locales contemplan, asimismo, un conjunto de líneas operativas dirigidas a la planificación de las infraestructuras urbanas al servicio de la dinamización empresarial. Aunque la diversidad de modalidades de intervención puede ser muy amplia, en esencia se agrupan en tres tipos de operaciones. El primero tiene como objetivo básico la mejora de los espacios industriales existentes, ya que frecuentemente los polígonos urbanos más antiguos se encuentran en franca situación de deterioro, están densamente congestionados y tienen una baja calidad urbanística, lo que les hace muy poco adecuados a las actuales necesidades de las empresas. De ahí, que se establezca el diseño y ejecución de planes de rehabilitación integral con el fin de mejorar sus condiciones ambientales, acondicionar adecuadamente sus infraestructuras y dotar de equipamientos y servicios a estos recintos industriales. La reordenación de las áreas fabriles heredadas se acompaña, en algunos casos, de programas de reubicación de instalaciones industriales desde las zonas más congestionadas hacia los nuevos espacios empresariales planificados.

Una segunda línea de actuación es la orientada a la ampliación y diversificación de la oferta de suelo urbanizado y bien comunicado para la instalación de nuevas empresas o para albergar las operaciones de traslado de instalaciones industriales desde otros sectores de la ciudad. También en este caso la tipología de la oferta que se pone en el mercado puede ser muy variada: suelo urbanizado para pymes, micropolígonos, polígonos para grandes empresas, áreas de actividad en las que coexisten parcelas de muy diverso tamaño para adaptarse con flexibilidad a la demanda empresarial, recintos empresariales para la integración de usos industriales y de servicios, etc. Se trata, en definitiva, de conseguir espacios económicos atractivos a través de la calidad del propio entorno empresarial y de sus características funcionales favoreciendo la consolidación y el crecimiento de las empresas existentes y la localización de nuevas actividades, lo que, en muchos casos, se facilita mediante la oferta de edificios industriales, naves con oficinas, naves-nido $u$ otras tipologías edificatorias de dimensiones y características muy variadas. Junto a este tipo de intervenciones, es cada vez más frecuente en las ciudades intermedias la creación de plataformas y áreas logísticas, parques empresariales y parques de oficinas y de negocios para diversificar la oferta de instalaciones y crear nuevas áreas de centralidad económica. 
La preparación o ampliación de las infraestructuras específicamente diseñadas para la instalación de actividades tecnológicamente avanzadas, constituye la tercera línea de actuación en la planificación del soporte físico para una economía urbana dinámica e innovadora. De nuevo, las tipologías en las que se concretan estas áreas y las denominaciones que reciben son muy diversas, pero básicamente se identifican con modalidades ya conocidas como son los parques tecnológicos (especializados o no en determinadas funciones, actividades o sectores), generalmente de iniciativa pública y con menor frecuencia de iniciativa privada o público-privada a través de consorcios o promotoras mixtas. Para evitar su carácter de enclave tecnológico con escasa inserción y débil repercusión en el tejido empresarial local, las actuaciones de promoción de los parques tecnológicos suelen encaminarse al establecimiento de vínculos entre las empresas innovadoras locales, la atracción de empresas externas, la integración de centros de investigación universitarios y centros tecnológicos públicos y privados y la organización de actividades y servicios de apoyo para la creación de redes de colaboración entre las propias unidades instaladas en el parque y entre éstas y el entramado empresarial de la ciudad de manera que el parque actúe como catalizador del potencial innovador local.

Integrados en los parques tecnológicos o vinculados a ellos, la promoción de centros de empresas e innovación, incubadoras, viveros y semilleros de empresas constituye, asimismo, otra de las opciones estratégicas en las políticas de dinamización urbana de las ciudades intermedias. Son centros que ofrecen locales en buenas condiciones (precio, instalaciones, servicios comunes...), infraestructuras de calidad, ambiente favorable y servicios de apoyo a los nuevos emprendedores, con el fin de estimular y facilitar el desarrollo de iniciativas empresariales nuevas y la creación de pymes innovadoras.

Menos numerosos hasta ahora pero proyectados ya en muchas ciudades intermedias son los parques científicos, espacios generalmente ubicados en el mismo campus universitario o en sus proximidades y destinados a albergar empresas innovadoras de base científica y departamentos de investigación, tanto universitarios como de otras instituciones públicas o de empresas privadas. En ellos se promueven las actividades de cooperación, la formación de redes y el desarrollo de vínculos entre los institutos tecnológicos, los equipos de investigación y los departamentos de I+D+i de las empresas para estimular las actividades punteras de investigación y la generación de conocimiento básico y aplicado orientado al desarrollo de nuevas actividades empresariales.

De forma complementaria a este tipo de espacios científico-tecnológicos, los planes estratégicos que se están implementando en algunas ciudades intermedias contemplan también otras actuaciones. Una sería la creación de nuevos espacios económicos digitales a partir de la transformación de zonas industriales tradicionales en espacios con una fuerte dotación de infraestructuras avanzadas, capaces de acoger proyectos empresariales de alto contenido tecnológico. Otra es la creación y el mantenimiento de infraestructuras inteligentes de soporte a la innovación — redes de telecomunicaciones, plataformas tecnológicas,...-, mediante el desarrollo de proyectos piloto basados en el uso intensivo de las nuevas tecnologías de la información y la comunicación (TIC) por parte de los ciudadanos, las empresas, las instituciones públicas y demás agentes sociales, culturales y económicos para extender posteriormente esta experiencia a todos los ámbitos de la ciudad. En otros casos, se trata de incentivar el alquiler de viviendas-incubadoras que, además de la residencia, proporcionen espacio para trabajar en el sector de los servicios avanzados o en las nuevas tecnologías; o bien de fomentar el desarrollo de este tipo de viviendas y actividades en los centros históricos como factor de revitalización urbana y construcción de redes de conocimiento entre los distintos grupos sociales de la ciudad. 


\subsection{La implicación de los agentes locales y el fortalecimiento del capital social}

En el proceso de elaboración de estrategias territoriales de innovación se requiere, en efecto, la participación activa de los agentes locales y la implicación e identificación de los ciudadanos con un proyecto común, de manera que orienten sus esfuerzos y actividades al logro de objetivos concretos. Además, la identificación participativa de los agentes (ciudadanos, empresas, entidades, instituciones...) con su territorio/espacio urbano se valora como un factor de cohesión social y un elemento de atracción y promoción de la ciudad. Es necesario para ello, que los poderes públicos ejerzan una intensa y efectiva labor de liderazgo y promuevan la participación social en el diseño de las dinámicas de innovación y en la formulación de las políticas urbanas. De ahí, que los documentos de planificación resalten el protagonismo político del gobierno municipal como verdadero impulsor y catalizador del dinamismo económico y social de la ciudad.

En esta línea de acción, las actuaciones públicas se encaminan a favorecer el fortalecimiento del capital social de la ciudad mediante la construcción de redes de proximidad socio-institucional y empresarial, y mediante el apoyo a la constitución de asociaciones público-privadas que colaboren de forma activa en el impulso de las innovaciones. Para ello, se precisa identificar y apoyar adecuadamente al conjunto de actores locales, formales e informales, que participan directa e indirectamente en los procesos de dinamización urbana - centros de innovación, empresas, cámaras de comercio, organismos de formación, emprendedores, asociaciones profesionales y culturales, instituciones universitarias... - para que desarrollen eficientemente su potencial y movilicen sus habilidades y activos. Se trataría, en suma, de promover un entramado social activo y ligado por lazos de confianza y reciprocidad y generadores de liderazgos que, a su vez, involucran la participación de la comunidad en proyectos comunes con la consiguiente creación de nuevo capital social

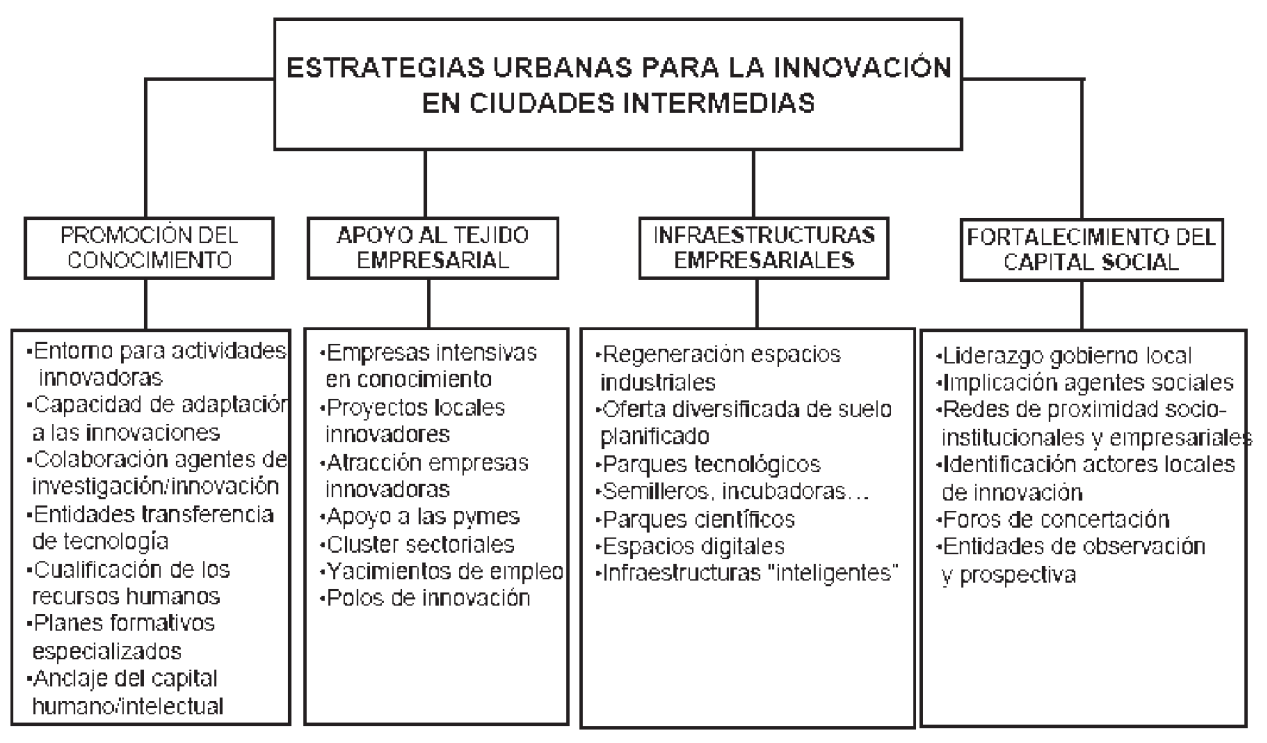

FIGURA 2. Estrategias urbanas para la innovación. Elaboración propia. 
territorial. A este fin se dirige la constitución de foros urbanos de concertación y participación ciudadana, y de coordinación de las iniciativas públicas — entre las administraciones locales y regionales - , así como, la creación de organismos/entidades de observación y prospectiva participados por los agentes sociales que responden, con denominaciones diversas - observatorios urbanos, económicos o industriales, barómetros urbanos, i-barómetros, radares empresariales, antenas tecnológicas...-, a objetivos y formas de organización y trabajo con ciertas analogías en las distintas ciudades.

\section{Consideraciones finales}

En suma, el análisis de las nuevas políticas urbanas refleja una profunda reorientación de los enfoques y prioridades en las estrategias de intervención pública, aunque también abre algunos interrogantes que no aparecen resueltos en los propios planes. De un lado, la proliferación de planes estratégicos y la repetición de objetivos e instrumentos muy similares entrañan, sin duda, el riesgo de generar una tendencia a la homogeneización que es precisamente contraria a la definición de la especificidad local - recursos territoriales específicos, activación del capital social... - como factor generador de ventajas competitivas. De otro, se aprecia una frecuente indefinición de los instrumentos y acciones concretas en los que se han de materializar las estrategias de activación de la innovación territorial con el fin de promover la competitividad urbana. Finalmente, el mayor protagonismo de los gobiernos locales en las políticas de innovación y desarrollo territorial generalmente no va acompañado de un mayor poder de decisión frente a las competencias de las administraciones regional y central. Buena parte de las actuaciones integradas en estas estrategias urbanas, desbordan ampliamente las competencias municipales e implican la intervención de instancias públicas que actúan a diferentes niveles y escalas. Esta superposición de instancias no siempre se resuelve de manera efectiva al agruparse en un mismo territorio actuaciones no coincidentes, o incluso en ocasiones contrapuestas, en sus objetivos y formas de intervención, lo que exige arbitrar mecanismos de coordinación mucho más eficaces en los que debe primar el interés general por encima de otras consideraciones.

\section{Bibliografía}

AGUADO, R. (2005): «Las nuevas herramientas de la política de innovación: los sistemas de innovación y el desarrollo de clusters», Boletín de Estudios Económicos, Vol. 60, n 186: 413-430.

ANDER-EGG, E. (1991): Introducción a la planificación. Ed. Siglo XXI, Madrid. 224 págs.

ARBONÍES ORTIZ, A. L. (2005): «Una propuesta conceptual sobre nuevos indicadores para la ciudad del conocimiento», Ekonomiaz, n ${ }^{\circ}$ 59: 232-245.

AREILZA, G. Y URRUTIA, V. M. (2000): «El poder local y las nuevas políticas urbanas», Documentación social $\mathrm{n}^{\circ}$ 119: 239-257.

BELLET, C. Y LLOP, J.M. (eds.) (2000): Ciudades intermedias. Urbanización y sostenibilidad. Editorial Milenio. Lleida. 562 págs.

BELLET, C. Y LLOP, J.M. (2004a): «Miradas a otros espacios urbanos. Las ciudades intermedias». Scripta Nova. Revista Electrónica de Geografía y Ciencias Sociales. Universidad de Barcelona. Vol. VIII, no 165: 1-30.

BELLET, C. Y LLOP, J.M. (2004b): «Ciudades intermedias: entre territorios concretos y ciudades y espacios globales». Ciudad y Territorio. Estudios Territoriales XXXVI, 141: 569-581.

BENITO, P. (2004): «Discursos, propuestas y acciones sobre la ciudad postindustrial». Anales de Geografía no 24: 9-29.

BOIX, R. (2003): Redes de ciudades y externalidades. Universitat Autònoma de Barcelona. http://www. tesisenxarxa.net/TESIS_UAB/AVAILABLE/TDX-0611104-145749//rbd1de4.pdf 
BOIX, R. (2006): «Redes de ciudades, economías externas y crecimiento», en A. Tarroja, A. y R. Camagni (coords.): Una nueva cultura del territorio. Criterios sociales y ambientales en las políticas y el gobierno del territorio, Diputació de Barcelona. Xarxa de Municipis, Barcelona: 251-263.

BORJA, J.; CASTELLS, M. (1997): Local y Global. La gestión de las ciudades en la era de la información. Ed. Taurus-United Nations for Human Settlements (Habitat), Madrid. 424 págs.

BRUGUÉ, J. Y GOMÀ R. (Coords.) (1998): Gobiernos locales y políticas públicas: bienestar social, promoción económica y territorio, Ariel, Barcelona.

BRUNET, R. (2000): «Des villes comme Lleida. Place et perspectives des villes moyennes en Europe», en C. Bellet, y J.M. Llop (eds.): Ciudades intermedias. Urbanización y sostenibilidad. Editorial Milenio. Lleida: 109-124.

CASTILLO HERMOSA, J. DEL Y DÍEZ FUENTE, C. (2006): «Infraestructuras de apoyo a la innovación científica y tecnológica: elementos clave de competitividad industrial», Ekonomiaz $n^{\circ}$ 63: 128-151.

COMISIÓN EUROPEA (1999): Estrategia Territorial Europea. Hacia un desarrollo equilibrado y sostenible del territorio de la UE. Comunidades Europeas, Luxemburgo.

COMISIÓN EUROPEA (2004): Tercer Informe sobre la Cohesión Económica y Social. Una nueva asociación para la cohesión. Convergencia, competitividad, cooperación. Comunidades Europeas, Luxemburgo.

COMISIÓN EUROPEA (2006): Política de cohesión y ciudades: la contribución urbana al crecimiento y el empleo en las regiones. Comunidades Europeas, Bruselas.

COMISIÓN EUROPEA (2007): Agenda Territorial de la Unión Europea. Hacia una Europa más competitiva y sostenible de regiones diversas. Comunidades Europeas, Luxemburgo.

COMISIÓN EUROPEA (2007): Informe general sobre la actividad de la Unión Europea 2006. Comunidades Europeas, Bruselas-Luxemburgo.

COMISIÓN EUROPEA (2007): The urban dimension in Community policies for the period 20072013. Comunidades Europeas, Bruselas.

ENGUITA PUEBLA, A. (1993): «Encuadre y método de la planificación estratégica», Urbanismo: Revista Oficial del COAM, n 19: 11-17.

ESPON (2005): Potentials for polycentric development in Europe. http://www.espon.eu/mmp/online/ website/content/projects/259/648/file_1174/fr-1.1.1_revised-full.pdf

ESPON (2006): The Role of Small and Medium-Size Towns (SMESTO). Final Report. http://www. espon.eu/mmp/online/website/content/projects/261/410/file_2225/fr-1.4.1_revised-full.pdf

ESPON (2007): Study on Urban Functions. Final Report. http://www.espon.eu/mmp/online/website/ content/projects/261/420/file_2420/fr-1.4.3_April2007-final.pdf

EUROPEAN UNION (2007): Regional Policy: State of European Cities Report. Adding Value to the European Urban Audit. http://ec.europa.eu/regional_policy/sources/docgener/studies/pdf/urban/ stateofcities_2007.pdf

EZQUIAGA, J.M. (2006): «Innovar, revitalizar, rehabilitar, reciclar: las bases de una nueva cultura urbanística, Análisis Local, nº 64: 55-58.

FERNÁNDEZ GÜELL, J. M. (1991): «Planificación estratégica de ciudades y territorios», en Muñiz, M.; Rodríguez, J. (eds.): Las grandes ciudades: debates y propuestas. Ed. Colegio de Economistas. Madrid: 285-290.

FERNÁNDEZ GÜELL, J. M. (2006): Planificación estratégica de ciudades. Ed. Reverté, Barcelona, 299 págs.

FORN, M. DE (1991): «Planificación estratégica y desarrollo económico. Su aplicación a Barcelona», en Muñiz, M.; Rodríguez, J. (eds.): Las grandes ciudades: debates y propuestas. Ed. Colegio de Economistas. Madrid: 291-311.

GANAU, J. Y VILAGRASA, J. (2003): «Ciudades medias en España: posición en la red urbana y procesos urbanos recientes», en H. Capel (coord.): Ciudades, arquitectura y espacio urbano. Instituto de Estudios Socioeconómicos de Cajamar. Almería: 37-73. http://www.fundacioncajamar. com/mediterraneo/revista/me0304.pdf

GASPAR, J. (2000): «Cidades médias e cidades intermédias. Novas funçoes territoriais e novas formas urbanas em Portugal», en C. Bellet, y J.M. Llop (eds.): Ciudades intermedias. Urbanización y sostenibilidad. Editorial Milenio. Lleida: 125-140. 
GÓMEZ RODRÍGUEZ, P.M. (2003): La gestión de marketing de ciudades y áreas metropolitanas: de la orientación al producto a la orientación al marketing, Cuadernos de Gestión, vol. 3, n ${ }^{\circ}$ 1-2: 11-26.

GONZÁLEZ ROMERO, G. (2006): «Innovación territorial y políticas públicas», Boletín de la A.G.E., $\mathrm{n}^{\circ}$ 42: 121-136.

HALL, P. (2000): «Redefining Europe's cities», en C. Bellet, y J.M. Llop (eds.): Ciudades intermedias. Urbanización y sostenibilidad. Editorial Milenio. Lleida: 93-108.

JUARISTI, J. (2000): «Sistemas urbanos y ciudades medias en el Arco Atlántico», en C. Bellet, y J.M. Llop (eds.): Ciudades intermedias. Urbanización y sostenibilidad. Editorial Milenio. Lleida: 143-167.

KEATING, M. (2005): «Gobernar las ciudades-región: política, economía y desarrollo», Ekonomiaz $\mathrm{n}^{\circ} 58: 128-145$.

MARTÍNEZ, M. (1999): «La traslación de estrategias empresariales al territorio: problemas de la Planificación Estratégica en el Urbanismo», Política y sociedad, 31, 93-116.

MELLA MÁRQUEZ, J.M. (1999): «Sobre la planificación estratégica de ciudades: algunas reflexiones», Análisis Local no 27: 11-18.

MÉNDEZ, R. (1995): «La industria en las nuevas políticas de desarrollo y ordenación en áreas metropolitanas». Anales de Geografía de la Universidad Complutense $\mathrm{n}^{\circ}$ 15: 451-463.

MÉNDEZ, R. (2001): «Innovación y redes de cooperación para el desarrollo local», Revista Internacional de Desenvolvimiento Local, Vol. 2, $\mathrm{n}^{\circ}$ 3: 37-44.

MÉNDEZ, R. (2002): «Innovación y desarrollo territorial: algunos debates teóricos recientes».Revista EURE, vol. XXVIII, $\mathrm{n}^{\circ}$ 84: 63-83.

MÉNDEZ, R. (2006): «Políticas de promoción y ordenación industrial en las áreas urbanas», en R. Méndez y H. Pascual (eds.): Industria y ciudad en España: nuevas realidades, nuevos retos. Thomson-Civitas, Cizur Menor: 51-74.

MÉNDEZ, R., MICHELINI, J.J. Y ROMEIRO, P. (2006): «Redes socio-institucionales e innovación para el desarrollo de las ciudades intermedias». Ciudad y Territorio Estudios Territoriales XXXVIII (148): 377-395.

MÉNDEZ, R. Y PASCUAL, H. (eds.) (2006): Industria y ciudad en España: nuevas realidades, nuevos retos. Thomson-Civitas, Cizur Menor. 618 págs.

MOULAERT, F. Y LAMBOY, J. G. (1998): «La organización económica de las ciudades: una perspectiva institucional», Ekonomiaz no 41: 118-147.

PARLAMENTO EUROPEO (2005): «Dimensión urbana en el contexto de la ampliación». Diario Oficial de la Unión Europea. 13/10/2005.

PASCUAL ESTEVE, J. Mª (2001): «De la planificación a la gestión estratégica de ciudades». Revista Elements de debat territorial, $\mathrm{n}^{\circ}$ 13: 4-45.

PASCUAL, J.M. Y FORN, M. DE (1999): La estrategia de las ciudades: los planes estratégicos como instrumento: métodos, técnicas y buenas prácticas, Barcelona, Diputació de Barcelona. 384 págs.

PRECEDO, A. (1993): «Las políticas de desarrollo y renovación urbana en Europa», Ciudad y Territorio. Estudios Territoriales $\mathrm{n}^{\circ}$ 98: 579-595.

RODRÍGUEZ, A., MOULAERT, F. Y SWINGEDOUW, E. (2001): «Nuevas políticas urbanas para la revitalización de las ciudades en Europa», Ciudad y territorio: Estudios territoriales $\mathrm{n}^{\circ}$ 129: 409-424.

SALOM, J. (2000): «El Arco Mediteráneo», en C. Bellet, y J.M. Llop (eds.): Ciudades intermedias. Urbanización y sostenibilidad. Editorial Milenio. Lleida: 209-223.

SPESP (2000): Study Programme on European Spatial Planning. Final Report. Brussels/Stockholm.

VÁZQUEZ BARQUERO, A. (1993): Política económica local. La respuesta de las ciudades a los desafíos del ajuste productivo. Ed. Pirámide. Madrid. 336 págs.

VILAGRASA, J. (1999): «Las ciudades pequeñas y medias en España», en R. Domínguez Rodríguez (coord.): La ciudad. Tamaño y crecimiento, Málaga, Universidad de Málaga y Asociación de Geógrafos Españoles: 17-50. 


\section{Planes Estratégicos citados}

Agencia Local de Promoción Económica y Empleo/Ayuntamiento de Gijón. II Plan Estratégico de Gijón. 2002-2012.

Ayuntamiento de Elche. Plan Estratégico Futurelx. Junio 2002.

Ayuntamiento de San Sebastián. Plan Estratégico de Donostia-San Sebastián. 2004.

Ayuntamiento de Valladolid. Valladolid hacia 2016. Propuestas Estratégicas. 2007.

Ayuntamiento de Vitoria-Gasteiz. Plan Estratégico Vitoria-Gasteiz 2010. La Estrategia para el Futuro de la Ciudad.

Burgos Ciudad 21. Asociación Plan Estratégico Ciudad de Burgos. Plan Estratégico de Burgos 2001-2015.

Córdoba Tercer Milenio. $2^{\circ}$ Plan Estratégico de Córdoba. Documento Central. La Estrategia Integrada Córdoba 2010. Octubre 2003.

Fundación Provigo. Plan Estratégico de Vigo y su Área Funcional. Plan Vigo 2010. Enero 2002.

Región de Murcia. Horizonte 2010. Plan Estratégico de la Región de Murcia 2007-2013. Julio 2006.

Segópolis. Plan Estratégico de Segovia y su área de influencia 2006-2016. 2006. 
